\title{
Order-disorder model of phase transitions in the DMAGaS-DMAAlS family crystals
}

\author{
I.V. Stasyuk and O.V. Velychko \\ Institute for Condensed Matter Physics \\ of the National Academy of Sciences of Ukraine, \\ 1 Svientsitskii Str., 79011 Lviv, Ukraine \\ e-mail: velychko@icmp.lviv.ua
}

\begin{abstract}
A four-state model is proposed for description of the sequence of phase transitions in ferroelectric crystals of DMAGaS and DMAAlS type. The ordering processes in the subsystems of DMA groups are considered as a main reason of such transformations. The interaction between groups in their various orientational states is taken into account in the dipole-dipole approximation. Obtained thermodynamical characteristics of the model (spontaneous polarization, occupancy of orientational states, dielectric susceptibility, phase diagram) are in good agreement with experimental data. The experimental fact of suppression of ferroelectric phase at increase of hydrostatic pressure is explained under assumption that pressure changes the difference between energies of various orientational states of DMA groups mostly.
\end{abstract}

PACS 77.84.-s, 64.60.Cn

Keywords: ferroelectrics, DMAAlS, DMAGaS, microscopic model

\section{Introduction}

A family of isomorphous crystals with ferroelectric properties to which e.g. $\left(\mathrm{CH}_{3}\right)_{2} \mathrm{NH}_{2} \mathrm{Al}\left(\mathrm{SO}_{4}\right)_{2} \cdot 6 \mathrm{H}_{2} \mathrm{O}(\mathrm{DMAAlS})$ and $\left(\mathrm{CH}_{3}\right)_{2} \mathrm{NH}_{2} \mathrm{Ga}\left(\mathrm{SO}_{4}\right)_{2} \cdot 6 \mathrm{H}_{2} \mathrm{O}$ (DMA$\mathrm{GaS}$ ) belong to is experimentally studied during the past decade. A peculiar feature of the family is a possible existence of the crystal in three different phases at change of temperature: at room temperature the crystal is paraelectric, at lowering of temperature it sequentially becomes ferroelectric and antiferroelectric (Pietraszko, Łukaszewicz and Kirpichnikova, 1993; Pietraszko and Łukaszewicz, 1994; Pietraszko, Łukaszewicz and Kirpichnikova, 1995; Tchukvinskyi, Cach and Czapla, 1998). For example, it has been measured $T_{c 1}=136 \mathrm{~K}$ and $T_{c 2}=113 \mathrm{~K}$ by Tchukvinskyi et al. (1998) (or $122 \mathrm{~K}$ and $114 \mathrm{~K}$ correspondingly (Pietraszko et al., 1995)) for DMAGaS 

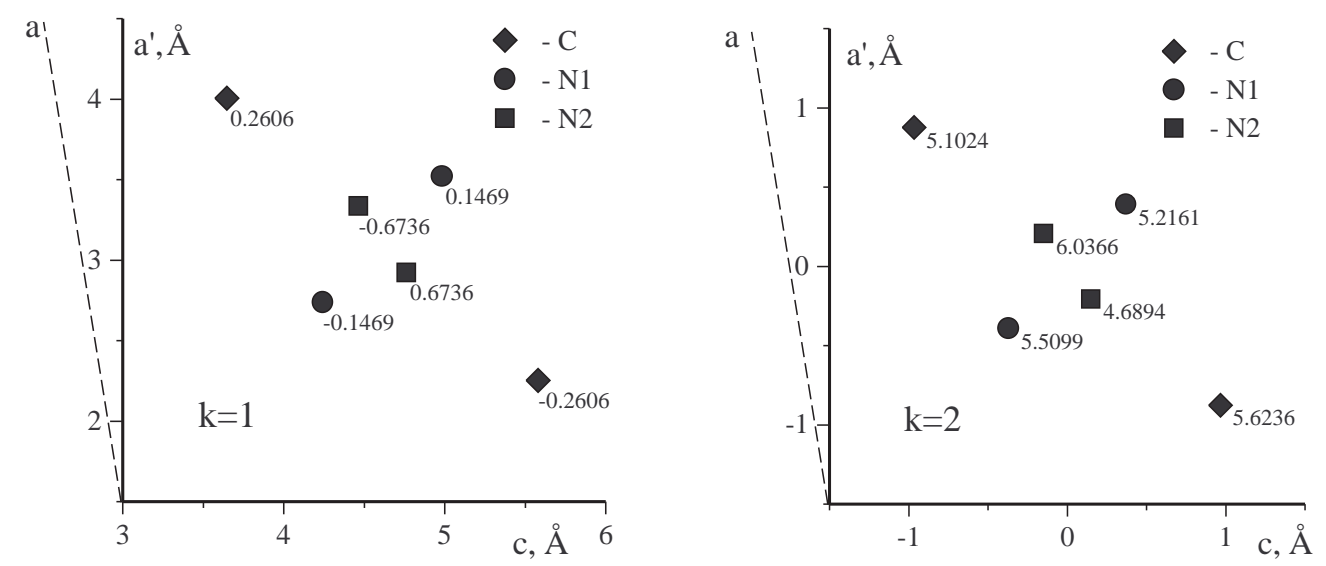

Figure 1: Projection of $\mathrm{N}$ and $\mathrm{C}$ atoms in the dimethylammonium groups of DMAGaS crystal at ambient temperature (Pietraszko et al., 1993) onto the ac plane (the $b$ coordinate is indicated for each atom; $k$ is the index of DMA sublattices).

crystal, but it has been only found $T_{c 1}=150 \mathrm{~K}$ (Pietraszko et al., 1993; Pietraszko et al., 1994; Pietraszko et al., 1995) for DMAAlS. The phase transition between the ferroelectric and antiferroelectric phases is of the first order (Pietraszko et al., 1995; Tchukvinskyi et al., 1998). There are evidences, supplied by optical, ultrasonic, pyroelectric, dilatometric and dielectric measurements, that the phase transition paraelectric - ferroelectric is of the first order close to the second one in DMAGaS and of the second order in DMAAlS. Crystallographic analysis shows that in all three phases the crystal belongs to monoclinic space groups: the high-temperature paraelectric phase has $\mathrm{P} 21 / \mathrm{n}$ space group (Pietraszko et al., 1993; Pietraszko et al., 1994), ferroelectric and antiferroelectric ones have Pn (Pietraszko et al., 1994; Pietraszko et al., 1995) and $\mathrm{P} 2_{1}$ (Pietraszko et al., 1995) groups respectively. It should be mentioned that low-symmetry space groups are subgroups of the highsymmetry group obtained by loss of rotation axis and mirror plane respectively (point symmetry group $2 / \mathrm{m}$ changes to $\mathrm{m}$ or 2 ).

Certain ferroelectric compounds have structural elements which reorientation leads to polarisation of the crystal. Changes in the rest of the crystal are insignificant and can be neglected in description of the phenomenon. Crystals DMAAlS and DMAGaS belong to this type of ferroelectrics. Here the element, which can be reoriented, is the dimethylammonium cation (more strictly $\mathrm{NH}_{2}$ group) (Pietraszko et al., 1995; Sobiestinskas, Grigas, Andreev and Varikash, 1992; Kazimirov et al., 1998; Dolinšek et al., 1999). This element can occupy four equilibrium positions related in pairs by inversion centre forming a slightly deformated cross (Figures [ 1 and 2). In the paraelectric phase a site in one pair $((k, 1)$ and $(k, 2) ; k=1,2)$ is occupied with probability $40 \%$ and in another $((k, 3)$ and $(k, 4))$ with probability $10 \%$ at $300 \mathrm{~K}$ (Pietraszko et al., 1993). Instead of the presented in Figure 1 obliqueangled crystallographic coordinates we shall use rectangular ones hereinafter. Their OY axes coincides with the $b$ axis, the OX axis is directed along the ferroelectric axis (N1-N1) and the OZ axis is chosen to make planes XZ and $a c$ identical.

According to the experimental data (Pietraszko et al., 1995) in the ferroelectric and antiferroelectric phases only positions in the first pair are occupied with 


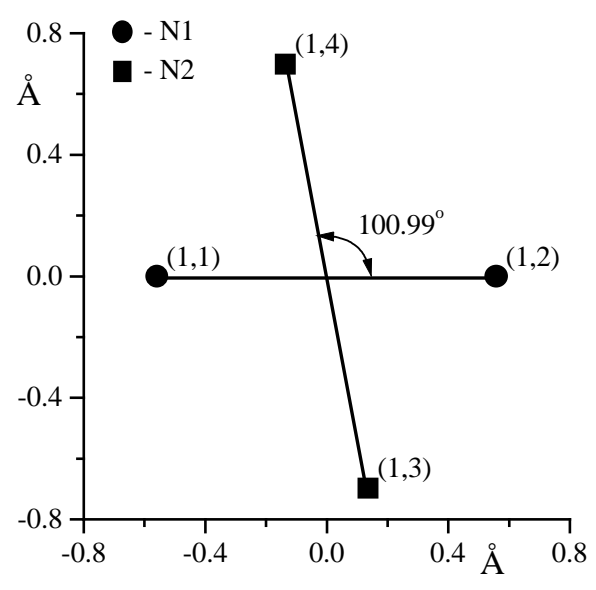

Figure 2: Projection of $\mathrm{N}$ atoms onto the plane perpendicular to the $\mathrm{C}-\mathrm{C}^{\prime}$ axis of the dimethylammonium group for the same crystal as in Figure 1.

Table 1: Conversion table of occupancies of orientational states into symmetrized ones which correspond to irreducible representations of the point symmetry group $2 / \mathrm{m}$.

\begin{tabular}{|c|c||c|c|c|c|c|c|c|c|}
\hline & & $(1,1)$ & $(1,2)$ & $(1,3)$ & $(1,4)$ & $(2,1)$ & $(2,2)$ & $(2,3)$ & $(2,4)$ \\
\hline \hline \multirow{2}{*}{$\mathrm{A}_{g}$} & $x_{+}$ & $1 / 2$ & $1 / 2$ & 0 & 0 & $1 / 2$ & $1 / 2$ & 0 & 0 \\
\cline { 2 - 10 } & $z_{+}$ & 0 & 0 & $1 / 2$ & $1 / 2$ & 0 & 0 & $1 / 2$ & $1 / 2$ \\
\hline \multirow{2}{*}{$\mathrm{B}_{g}$} & $x_{-}$ & $1 / 2$ & $1 / 2$ & 0 & 0 & $-1 / 2$ & $-1 / 2$ & 0 & 0 \\
\cline { 2 - 10 } & $z_{-}$ & 0 & 0 & $1 / 2$ & $1 / 2$ & 0 & 0 & $-1 / 2$ & $-1 / 2$ \\
\hline \multirow{2}{*}{$\mathrm{B}_{u}$} & $y_{+}$ & $1 / 2$ & $-1 / 2$ & 0 & 0 & $1 / 2$ & $-1 / 2$ & 0 & 0 \\
\cline { 2 - 10 } & $u_{-}$ & 0 & 0 & $1 / 2$ & $-1 / 2$ & 0 & 0 & $-1 / 2$ & $1 / 2$ \\
\hline \multirow{2}{*}{$\mathrm{A}_{u}$} & $y_{-}$ & $1 / 2$ & $-1 / 2$ & 0 & 0 & $-1 / 2$ & $1 / 2$ & 0 & 0 \\
\cline { 2 - 10 } & $u_{+}$ & 0 & 0 & $1 / 2$ & $-1 / 2$ & 0 & 0 & $1 / 2$ & $-1 / 2$ \\
\hline
\end{tabular}

appropriate ordering.

In the ferroelectric phase positions $(1,2)$ and $(2,2)$ (or $(1,1)$ and $(2,1)$ ) are mainly occupied while in the antiferroelectric state occupancies of positions $(1,2)$ and $(2,1)$ (or $(1,1)$ and $(2,2)$ respectively) prevail (Pietraszko et al., 1993). In (Stasyuk and Velychko, 2000a) we have performed the symmetry analysis of functions being linear combinations of position occupancies of $\mathrm{NH}_{2}$ groups in an elementary cell. Symmetrized linear combinations transforming according to irreducible representations of point symmetry group $2 / \mathrm{m}$ of the high-temperature phase are presented in Table 1. Combinations $y_{+}, u_{-}\left(y_{-}, u_{+}\right)$belonging to representation $B_{u}\left(A_{u}\right)$ correspond to orderings appearing in the ferroelectric (antiferroelectric) phase and form order parameters for these phases. Existing orderings are of a mixed nature: ferroelectric ordering in positions $(k, 1)$ and $(k, 2)$ (along the OX axis) is accompanied by antiferroelectric one in $(k, 3)$ and $(k, 4)$ (along the OY axis) and vice versa.

Proceeding from these facts we have proposed microscopic four-state model (Sta- 
syuk et al., 2000a) for description of thermodynamics of order-disorder phase transitions in the subsystem of reorientationable DMA groups. The phase transition between the paraelectric and ferroelectric phases in the considered crystals was described and criteria of order of this transition were established in the approach of a direct interaction between variously oriented groups (a site-site approach). The results obtained can not be extended on a wide temperature range including e.g. the region of the low-temperature phase transition in DMAGaS because total occupancies of "longitudinal" positions 1 and 2 (and "transverse" positions 3 and 4 respectively) have been assumed as temperature independent parameters of theory. Nevertheless in this work order parameters corresponding to anti- and ferroelectric orderings have been constructed what allowed to describe the phase transition between ferro- and paraelectric phases and to establish criteria of its order.

Here a continuation of this work is presented where an approach of dipole-dipole interaction between ionic groups is used; temperature change of total occupancies is taken into account as well as the effect of reorientational hopping of groups between positions $(k, p)$. Ordering of ionic groups corresponding to order parameters of both $B_{u}$ and $A_{u}$ symmetry is described in the mean field approximation. Equilibrium states are investigated, temperature dependences of order parameters (spontaneous polarization) and occupancies of positions of $\mathrm{NH}_{2}$ groups are studied. Both phase transitions (at temperatures $T_{c 1}$ and $T_{c 2}$ ) are described; the regions of existence of para-, ferro- and antiferroelectric phases depending on temperature and model parameters are established. Observed by experiment changes of picture of phase transitions in DMAGaS crystal under the influence of hydrostatic pressure are interpreted in the framework of microscopic description.

\section{Hamiltonian of the model}

Let us formulate Hamiltonian of the subsystem of DMA groups on the basis of four orientational states with taking into account of differences of energies of nonequivalent configurations, possibility of orientational hopping and interaction between groups in dipole approximation.

$$
H=-\sum_{n k} \sum_{\alpha} E_{\alpha} D_{n k}^{\alpha}+\sum_{n k} \sum_{s s^{\prime}} \lambda_{k}^{s s^{\prime}} X_{n k}^{s s^{\prime}}-\frac{1}{2} \sum_{n n^{\prime}} \sum_{k k^{\prime}} \sum_{\alpha \beta} \Psi_{\alpha \beta}^{k k^{\prime}}\left(n n^{\prime}\right) D_{n k}^{\alpha} D_{n^{\prime} k^{\prime}}^{\alpha^{\prime}},
$$

where

$$
\begin{aligned}
& \hat{\lambda}_{1}=\left(\begin{array}{cccc}
\varepsilon_{1} & 0 & \Omega_{1} & \Omega_{2} \\
0 & \varepsilon_{1} & \Omega_{2} & \Omega_{1} \\
\Omega_{1} & \Omega_{2} & \varepsilon_{2} & 0 \\
\Omega_{2} & \Omega_{1} & 0 & \varepsilon_{2}
\end{array}\right), \quad \hat{\lambda}_{2}=\left(\begin{array}{cccc}
\varepsilon_{1} & 0 & \Omega_{2} & \Omega_{1} \\
0 & \varepsilon_{1} & \Omega_{1} & \Omega_{2} \\
\Omega_{2} & \Omega_{1} & \varepsilon_{2} & 0 \\
\Omega_{1} & \Omega_{2} & 0 & \varepsilon_{2}
\end{array}\right), \\
& D_{n k}^{x}=d_{x}\left(X_{n k}^{22}-X_{n k}^{11}\right), \quad D_{n k}^{y}=d_{y}\left(X_{n k}^{44}-X_{n k}^{33}\right), \quad D_{n k}^{z}=0,
\end{aligned}
$$

$X_{n k}^{s s^{\prime}}$ is the Hubbard operator describing transition from the state $s^{\prime}$ on the state $s$ of the DMA complex in the lattice site $n$ and the sublattice $k\left(X_{n k}^{s s^{\prime}}=|n k, s\rangle\left\langle n k, s^{\prime}\right|\right)$, $D_{n k}^{\alpha}$ is the $\alpha$-component of the dipole moment of complex, $E_{\alpha}$ is the corresponding component of external electric field, $\Psi_{\alpha \beta}^{k k^{\prime}}\left(n n^{\prime}\right)$ is the energy of the dipole interaction, 
$\varepsilon_{1}$ and $\varepsilon_{2}$ are the energies of DMA groups in positions $(k, 1),(k, 2)$ and $(k, 3),(k, 4)$ respectively, $\Omega_{1}$ and $\Omega_{2}$ are the parameters of the reorientational hopping.

Here the coordinate system with the $\mathrm{X}$ axis along the axis of spontaneous polarization of the crystal and the $\mathrm{Y}$ axis perpendicular to the DMA plane (parallel to the crystallographic $b$ axis) is used.

It should be mentioned that the Hamiltonian (11) simplifies the real structure of DMA complexes in DMAGaS crystals, which are oriented not exactly along the chosen axes (as assumed in definitions of $D_{n k}^{\alpha}($ (3) ) ). Next, in our case the dipoledipole interaction is only the main term of the multipole expansion. Nevertheless, as will be shown below, the model described by the Hamiltonian (1) can give a satisfactory explanation of the behaviour of DMAGaS and DMAAlS-type crystals despite of simplifications made.

The Hamiltonian (1) can be rewritten to separate the mean field part

$$
\begin{aligned}
H & =N H_{\mathrm{C}}+H_{\mathrm{MF}}+H^{\prime}, \\
H_{\mathrm{C}} & =\frac{1}{2} \sum_{k k^{\prime}} \sum_{\alpha \beta} \psi_{\alpha \beta}^{k k^{\prime}}\left\langle D_{k}^{\alpha}\right\rangle\left\langle D_{k^{\prime}}^{\beta}\right\rangle, \\
H_{\mathrm{MF}} & =\sum_{n k} \sum_{s s^{\prime}} \lambda_{k}^{s s^{\prime}} X_{n k}^{s s^{\prime}}-\sum_{n k} \sum_{\alpha}\left(F_{\alpha}^{k}+E_{\alpha}\right) D_{n k}^{\alpha},
\end{aligned}
$$

where

$$
F_{\alpha}^{k}=\sum_{k^{\prime}} \sum_{\beta} \psi_{\alpha \beta}^{k k^{\prime}}\left\langle D_{k^{\prime}}^{\beta}\right\rangle, \quad \psi_{\alpha \beta}^{k k^{\prime}}=\sum_{n^{\prime}} \Psi_{\alpha \beta}^{k k^{\prime}}\left(n n^{\prime}\right)
$$

$N$ is the total number of sites. Further considerations will be restricted to the mean field approximation (MFA) and hence the fluctuative term $H^{\prime}$ is neglected.

It is convenient to make a unitary transformation to linear combinations of dipole moments

$$
\left\langle D_{\eta}\right\rangle=\sum_{k \alpha} U_{\eta, k \alpha}\left\langle D_{k}^{\alpha}\right\rangle
$$

transforming according to the irreducible representations of the high-temperature symmetry group of the crystal (cf. Appendix A). After that the MFA Hamiltonian looks like

$$
\begin{aligned}
H_{\mathrm{MFA}} & =N H_{\mathrm{C}}+H_{\mathrm{MF}}, \\
H_{\mathrm{C}} & =\frac{1}{2} \sum_{\eta \eta^{\prime}}\left\langle\tilde{D}_{\eta}\right\rangle \psi_{\eta \eta^{\prime}}\left\langle\tilde{D}_{\eta^{\prime}}\right\rangle \\
H_{\mathrm{MF}} & =\sum_{n k} \sum_{s s^{\prime}} B_{k}^{s s^{\prime}} X_{n k}^{s s^{\prime}}
\end{aligned}
$$

where

$$
\begin{aligned}
& B_{k}^{s s^{\prime}}=\lambda_{k}^{s s^{\prime}}-\delta_{s s^{\prime}} \sum_{\eta}\left(\sum_{\alpha} U_{\eta, k \alpha} d_{k \alpha}^{s s}\right)\left(\tilde{F}_{\eta}+\tilde{E}_{\eta}\right) \\
& \tilde{F}_{\eta}=\sum_{\eta^{\prime}} \psi_{\eta \eta^{\prime}}\left\langle\tilde{D}_{\eta^{\prime}}\right\rangle, \quad \psi_{\eta \eta^{\prime}}=\sum_{k k^{\prime}} \sum_{\alpha \alpha^{\prime}} U_{\eta, k \alpha} \psi_{k k^{\prime}}^{\alpha \alpha^{\prime}} U_{\eta^{\prime}, k^{\prime} \alpha^{\prime}}
\end{aligned}
$$




$$
\begin{aligned}
& \tilde{E}_{\eta}=\sum_{k \alpha} U_{\eta, k \alpha} E_{\alpha}, \quad \tilde{E}_{1}=\tilde{E}_{3}=\tilde{E}_{5}=0, \\
& \tilde{E}_{2}=\sqrt{2} E_{y}, \tilde{E}_{4}=\sqrt{2} E_{x}, \tilde{E}_{6}=\sqrt{2} E_{z} .
\end{aligned}
$$

Symmetrized averages of dipole moments form two pairs belonging to different irreducible representations:

$$
\begin{array}{lll}
A_{u}: & \left\langle\tilde{D}_{1}\right\rangle=\left(\left\langle D_{1}^{x}\right\rangle-\left\langle D_{2}^{x}\right\rangle\right) / \sqrt{2}, & \left\langle\tilde{D}_{2}\right\rangle=\left(\left\langle D_{1}^{y}\right\rangle+\left\langle D_{2}^{y}\right\rangle\right) / \sqrt{2} \\
B_{u}: & \left\langle\tilde{D}_{4}\right\rangle=\left(\left\langle D_{1}^{x}\right\rangle+\left\langle D_{2}^{x}\right\rangle\right) / \sqrt{2}, & \left\langle\tilde{D}_{5}\right\rangle=\left(\left\langle D_{1}^{y}\right\rangle-\left\langle D_{2}^{y}\right\rangle\right) / \sqrt{2}
\end{array}
$$

Matrix $\psi_{\eta \eta^{\prime}}$ has a block-diagonal structure with nonzero blocks belonging to the given representation (see Appendix A).

As follows from the expressions (12) and (13) the antiferroelectric ordering along the $\mathrm{X}$ axis is accompanied by the ferroelectric one along the $\mathrm{Y}$ axis and vice versa.

In general case $\Omega_{i} \neq 0$ the diagonalization procedure should be applied to the matrix $\hat{B}_{k}$ in the expression (10):

$$
H_{\mathrm{MF}}=\sum_{r} \tilde{\lambda}_{k}^{r} \tilde{X}_{n k}^{r r}
$$

where

$$
\tilde{\lambda}_{k}^{r}=\sum_{s s^{\prime}} V_{s r}^{k} B_{k}^{s s^{\prime}} V_{s^{\prime} r}^{k}, \quad \tilde{X}_{n k}^{r r}=\sum_{s s^{\prime}} V_{s r}^{k} X_{n k}^{s s^{\prime}} V_{s^{\prime} r}^{k}
$$

and

$$
\left\langle D_{k}^{\alpha}\right\rangle=\sum_{r} \tilde{d}_{k \alpha}^{r r}\left\langle\tilde{X}_{k}^{r r}\right\rangle, \quad \tilde{d}_{k \alpha}^{r r}=\sum_{s s^{\prime}} V_{s r}^{k} d_{\alpha}^{s s^{\prime}} V_{s^{\prime} r}^{k}
$$

Now all necessary thermodynamical functions could be derived from the Hamiltonian (8) with corresponding terms expressed by (9) and (14).

\section{Thermodynamics of the model}

The partition function of the model in general form is presented as

$$
Z=Z_{1}^{N} Z_{2}^{N} \exp \left(-\beta N H_{\mathrm{C}}\right), \quad \beta=1 / k_{\mathrm{B}} T
$$

where

$$
\begin{aligned}
Z_{k}= & \sum_{s} \exp \left(-\beta \tilde{\lambda}_{k}^{s}\right), \quad k=1,2 \\
H_{\mathrm{C}}= & \frac{1}{2}\left(a_{1}\left\langle\tilde{D}_{1}\right\rangle^{2}+d_{1}\left\langle\tilde{D}_{2}\right\rangle^{2}+2 b_{1}\left\langle\tilde{D}_{1}\right\rangle\left\langle\tilde{D}_{2}\right\rangle\right. \\
& \left.\quad+a_{2}\left\langle\tilde{D}_{4}\right\rangle^{2}+d_{2}\left\langle\tilde{D}_{5}\right\rangle^{2}+2 b_{2}\left\langle\tilde{D}_{4}\right\rangle\left\langle\tilde{D}_{5}\right\rangle\right) .
\end{aligned}
$$

As a result free energy per particle looks like

$$
F=-\frac{1}{\beta N} \ln Z=H_{\mathrm{C}}-\frac{1}{\beta} \ln Z_{1}-\frac{1}{\beta} \ln Z_{2} .
$$


Now one can calculate averages

$$
\left\langle X_{k}^{r r}\right\rangle=\exp \left(-\beta \tilde{\lambda}_{k}^{r}\right) / \sum_{s} \exp \left(-\beta \tilde{\lambda}_{k}^{s}\right)
$$

and after substitution into formulae (12), (13) and (16) selfconsistency equations for $\left\langle\tilde{D}_{n}\right\rangle$ can be obtained.

In the case $\Omega_{1}=\Omega_{2}=0$ the matrix $\hat{B}_{k}$ is diagonal and expressions for thermodynamical functions are more simple. For example, the set of selfconsistency equations looks like

$$
\begin{aligned}
& \left\langle\tilde{D}_{1,4}\right\rangle=\frac{d_{x}}{\sqrt{2}} \exp (\beta \Delta)\left[\mathcal{Z}_{1}^{-1} \sinh \left(\beta \varkappa_{1}^{x}\right) \mp \mathcal{Z}_{2}^{-1} \sinh \left(\beta \varkappa_{2}^{x}\right)\right] \\
& \left\langle\tilde{D}_{2,5}\right\rangle=\frac{d_{y}}{\sqrt{2}} \exp (-\beta \Delta)\left[\mathcal{Z}_{1}^{-1} \sinh \left(\beta \varkappa_{1}^{y}\right) \pm \mathcal{Z}_{2}^{-1} \sinh \left(\beta \varkappa_{2}^{y}\right)\right]
\end{aligned}
$$

where

$$
\begin{aligned}
\mathcal{Z}_{k}= & \exp (\beta \Delta) \cosh \left(\beta \varkappa_{k}^{x}\right)+\exp (-\beta \Delta) \cosh \left(\beta \varkappa_{k}^{y}\right), \quad k=1,2, \\
\varkappa_{k}^{x}= & \frac{d_{x}}{\sqrt{2}}\left[(-1)^{\delta_{k, 2}}\left(a_{1}\left\langle\tilde{D}_{1}\right\rangle+b_{1}\left\langle\tilde{D}_{2}\right\rangle\right)+a_{2}\left\langle\tilde{D}_{4}\right\rangle+b_{2}\left\langle\tilde{D}_{5}\right\rangle+\sqrt{2} E_{x}\right], \\
\varkappa_{k}^{y}= & \frac{d_{y}}{\sqrt{2}}\left[b_{1}\left\langle\tilde{D}_{1}\right\rangle+d_{1}\left\langle\tilde{D}_{2}\right\rangle+(-1)^{\delta_{k, 2}}\left(b_{2}\left\langle\tilde{D}_{4}\right\rangle+d_{2}\left\langle\tilde{D}_{5}\right\rangle\right)+\sqrt{2} E_{y}\right], \\
& \varepsilon=\left(\varepsilon_{2}+\varepsilon_{1}\right) / 2, \quad \Delta=\left(\varepsilon_{2}-\varepsilon_{1}\right) / 2 .
\end{aligned}
$$

Components of static dielectric susceptibility are determined in usual way

$$
\begin{aligned}
& \chi_{x}=\frac{\partial P_{x}}{\partial E_{x}}=\frac{1}{v_{c}} \frac{\partial\left\langle D_{1}^{x}+D_{2}^{x}\right\rangle}{\partial E_{x}}=\frac{\sqrt{2}}{v_{c}} \frac{\partial\left\langle\tilde{D}_{4}^{x}\right\rangle}{\partial E_{x}}, \\
& \chi_{y}=\frac{\partial P_{y}}{\partial E_{y}}=\frac{1}{v_{c}} \frac{\partial\left\langle D_{1}^{y}+D_{2}^{y}\right\rangle}{\partial E_{y}}=\frac{\sqrt{2}}{v_{c}} \frac{\partial\left\langle\tilde{D}_{2}^{x}\right\rangle}{\partial E_{y}},
\end{aligned}
$$

where $P_{x}$ and $P_{y}$ are the components of spontaneous polarization and $v_{c}$ is the volume of an elementary cell. After straightforward but cumbersome calculations one can derive them from set of equations (22) in the explicit form

$$
\begin{aligned}
& \chi_{x}=\frac{1}{v_{c}} \frac{2\left[\Theta K_{x}-d_{2}\left(K_{x} K_{y}-L^{2}\right)\right]}{\Theta^{2}+\Theta\left(2 b_{2} L-a_{2} K_{x}-d_{2} K_{y}\right)+\left(a_{2} d_{2}-b_{2}^{2}\right)\left(K_{x} K_{y}-L^{2}\right)}, \\
& \chi_{y}=\frac{1}{v_{c}} \frac{2\left[\Theta K_{y}-a_{1}\left(K_{x} K_{y}-L^{2}\right)\right]}{\Theta^{2}+\Theta\left(2 b_{1} L-a_{1} K_{x}-d_{1} K_{y}\right)+\left(a_{1} d_{1}-b_{1}^{2}\right)\left(K_{x} K_{y}-L^{2}\right)},
\end{aligned}
$$

where

$$
\begin{aligned}
& K_{\alpha}=d_{\alpha}^{2} N_{\alpha}-\left\langle D_{1}^{\alpha}\right\rangle^{2}, \quad \alpha=x, y ; \quad L=\left\langle D_{1}^{x}\right\rangle\left\langle D_{1}^{y}\right\rangle \\
& N_{x}=\left\langle X^{11}+X^{22}\right\rangle, \quad N_{y}=\left\langle X^{33}+X^{44}\right\rangle, \quad \Theta=k_{\mathrm{B}} T .
\end{aligned}
$$




\section{Numerical results}

The model under consideration demonstrates a complicated thermodynamical behaviour and a some numbers of qualitatively different phase sequences dependently on parameter values. Further study will be devoted to the case of the sequence of phase transitions which is characteristic to the DMAGaS crystal (antiferroelectric $\rightarrow$ ferroelectric $\rightarrow$ paraelectric phases with temperature increase).

Such a sequence of phase transition is achieved at the next relationships between parameters

$$
\begin{aligned}
& 0<b_{1}, d_{1}, a_{1}<b_{2}, d_{2} \\
& a_{1}>a_{2}>0
\end{aligned}
$$

If the above nonequalities are fulfilled, antiparallel orientation of $D_{x^{-}}$and $D_{y}$-dipoles of neighbour DMA groups is energetically favourable. At low temperatures (when occupancies of positions $(k, 3)$ and $(k, 4)$ tend to zero) the antiferroelectric phase related with antiparallel dipoles $D_{x}$ is realized. In the result of increased probability of appearance of groups in the mentioned positions at temperature growing the interaction with participation of $D_{y}$ dipoles becomes significant. The phase transition to the phase with their antiferroelectric orientation occurs when respective interactions become prevailing. This phase is simultaneously ferroelectric along the OX axis.

There are two other more subtle conditions: for a correct description of the DMAGaS crystal the high-temperature phase transition should be of the first order and the ratio $\left(\Theta_{c 1}-\Theta_{c 2}\right) / \Theta_{c 1}$ should correspond to the experimental one.

Results of numerical calculations of temperature dependences of symmetrized dipole moments (12), (13) (obtained as solutions of equation set (22)) having meaning of order parameters are depicted in Figure 3. Here the case of the first order high-temperature phase transition close to the second one is illustrated. Besides the temperatures of real phase transitions $\Theta_{c 1}$ and $\Theta_{c 2}$, the temperature of a possible phase transition $\Theta_{c}^{\prime}$ (which might be realized in the absence of the ferroelectric phase) is indicated.

As one can see in Figure 3 and from the expressions (12), (13) antiferroelectric orientation along the $\mathrm{X}$ axis is accompanied by ferroelectric one along the $\mathrm{Y}$ axis and vice versa. Polarization along the $\mathrm{X}$ axis is larger than along the $\mathrm{Y}$ one due to larger occupancies of positions 1 and 2 comparatively to positions 3 and 4 (Figure 4).

Behaviour of the spontaneous polarisation $P_{x}$ (which is proportional to $\left\langle\tilde{D}_{4}\right\rangle$ as stated in (23)) strictly corresponds to experimental measurements (Dacko and Czapla, 1996; Pykacz and Czapla, 1997). But despite of common assumption (see e.g. Kazimirov et al., 1998) at lowering of temperature the occupancy of the positions 3 and 4 do not tend to zero monotonically: occupancies are asymmetrical and $\left\langle X_{1}^{44}\right\rangle$ even increases in the ferroelectric phase (with vice versa occupancies for $k=2$ ).

The influence of the orientational hopping on the phase transition in the system resemble one for Ising model (Figure 5 - for simplicity is assumed $\Omega_{1}=\Omega_{2}=\Omega$ ). Namely the increase of $\Omega$ value leads as a whole to the decrease of temperatures of both phase transitions and their sequential zeroing at corresponding values $\Omega_{c 2}$ and $\Omega_{c 1}$. Antiferroelectric phase vanishes at relatively low values of $\Omega$ when the 

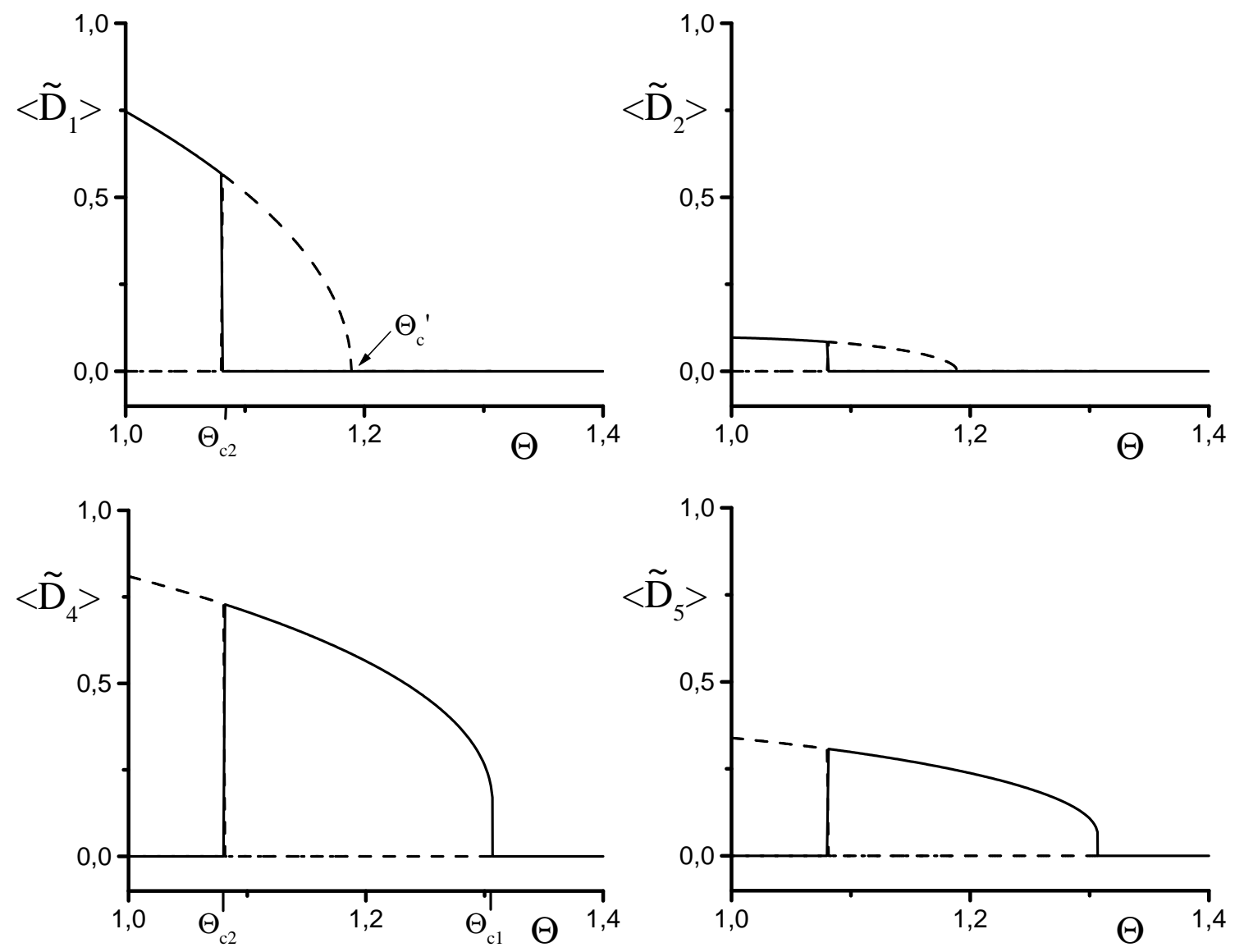

Figure 3: Dependences of $\langle\tilde{D}\rangle$ on temperature at specified below values of parameters: $b_{1} / a_{1}=1.55, d_{1} / a_{1}=0.5, a_{2} / a_{1}=0.4, b_{2} / a_{1}=2.6, d_{2} / a_{1}=1.6, \Delta / a_{1} d_{x}^{2}=1.5855$, $\Omega_{1}=\Omega_{1}=0, d_{y} / d_{x}=1.4 ; \Theta$ is given in $a_{1} d_{x}^{2}$ units. The same values of parameters are held in Figures 4 8. Solid lines correspond to the thermodynamically stable solution, dashed and dotted - to the unstable ones. 

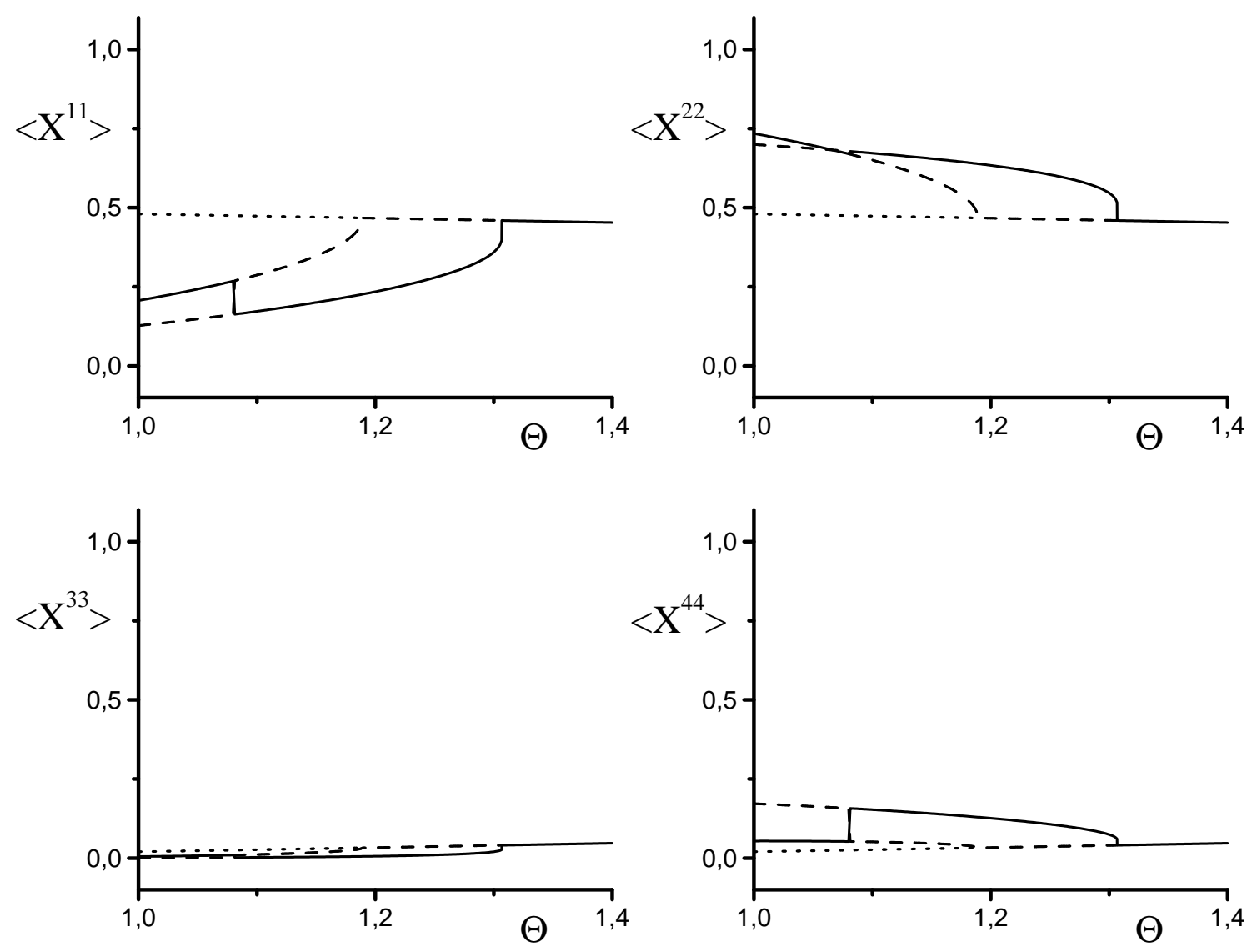

Figure 4: Dependences of occupancies of states on temperature. Here $k=1$.
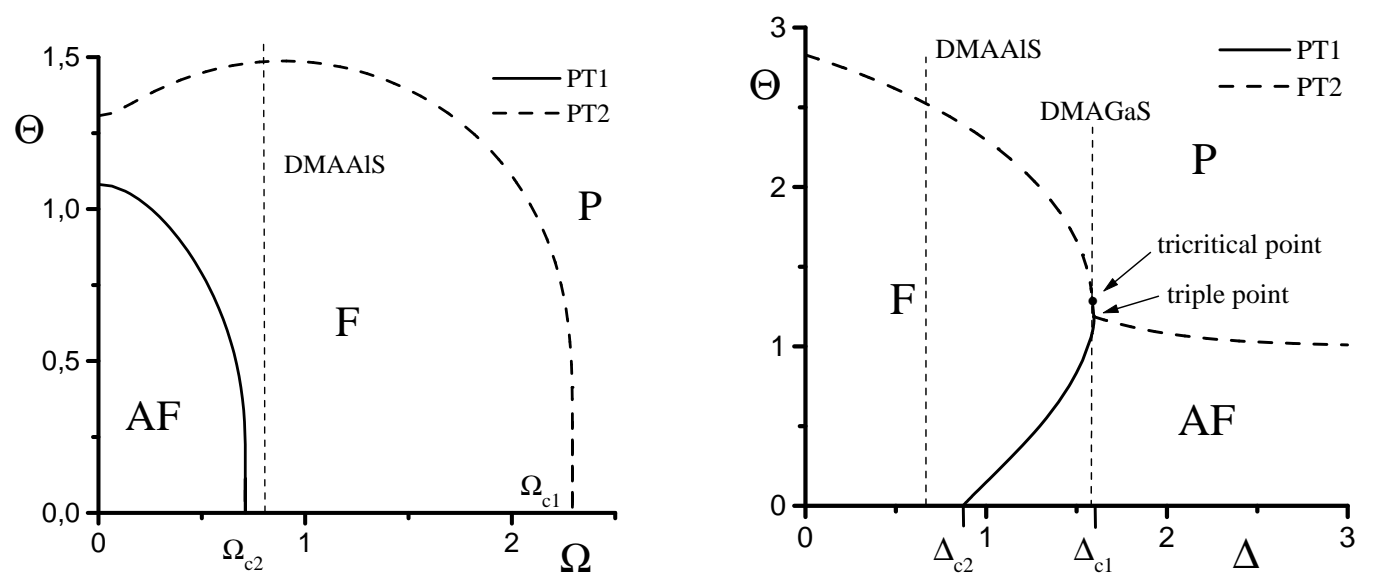

Figure 5: Phase diagram $\Theta-\Omega . \Theta$ and Figure 6: Phase diagram $\Theta-\Delta . \Theta$ and $\Omega$ are given $a_{1} d_{x}^{2}$ units. $\Delta$ are given $a_{1} d_{x}^{2}$ units. 
temperature of the other phase transition changes insignificantly. Due to prevailing suppression of antiferroelectric ordering and the mentioned above competition between anti- and ferroelectric interactions the considered diagram has a peculiar feature: the temperature of the ferroelectric-paraelectric phase transition increases at small value of $\Omega$.

According to the experimental data on pressure effects in DMAGaS (Yasuda, Kaneda and Czapla, 1999) applying and increase of hydrostatic pressure leads to the narrowing of the temperature region of existence of the ferroelectric phase. At certain pressure the ferroelectric phase is completely suppressed. Thermodynamical description in the framework of the Landau theory allows to reproduce observed phase diagram by setting coefficients at the second order terms in the Landau expansion of free energy to be pressure dependent (Stasyuk, Velychko, Czapla and Czukwinski, 2000b). Microscopic description of influence of hydrostatic pressure is rather a complicated question. One can assume, that due to limited compressibility of the crystal interaction constants change insignificantly and the main effect is due to the increase of the difference of site energies $\Delta$. In this case the obtained phase diagram remarkably good coincides with qualitative form of the experimental one (Figure 6). All three phases could exist at change of temperature in the region bounded by values $\Delta_{c 1}$ and $\Delta_{c 2}$. This confirms the above assumption of prevailing influence of pressure on site energies. Nevertheless, it seems that a more complete reproduction of the observed dependences (in particular, the increase of temperature of phase transition in the antiferroelectric phase at the pressure values above the triple point) can be achieved when additional factors (such as change of interaction constants or the parameter $\Omega$ ) will be taken into account. For example, values of parameters of interaction are known to increase at rising of pressure. The temperature $\Theta$ presented in Figures 3 - 8 is made dimensionless by division on $a_{1} d_{x}^{2}$. Hence if the dimensionless $\Theta_{c}$ remains constant at increase of pressure, the real temperature of the phase transition grows up.

As one can mention there are both triple and tricritical points on the diagram. There is a narrow region of $\Delta$ values near the triple point where both phase transition are of the first order as in the case of DMAGaS crystals.

Parameters in Figures 3 and 4 are chosen to fulfill the mentioned above conditions and to give the best description of experimental data for DMAGaS crystal. On the other hand, a typical behaviour of the DMAAlS crystal could be obtained in two different way. One can assume that value of the parameter $\Delta$ is smaller than $\Delta_{c 2}$ for this case (Figure 6). Alternatively, value of the parameter $\Omega$ can be less than $\Omega_{c 2}$ (Figure 5). In both cases it gives direct transition of the second order from the paraelectric phase to the ferroelectric one at lowering of temperature.

Dependences of inverse components of dielectric susceptibility are presented in Figure 7. $\chi_{x}(T)$ has a pronounced peak at the temperature of phase transition between ferroelectric and paraelectric phases but does not reach singularity. Such a behaviour good coincides with the experimental measurements of dielectric permittivity made by Tchukvinsky et al. (1998). Due to the first order of the hightemperature phase transition the slope of the $\chi_{x}(T)$ curve changes more than twice in the phase transition point. $\chi_{y}(T)$ has a small peak in this point (Figure 8). There is a lack of experimantal data considering dielectric measurements along the OY axis 

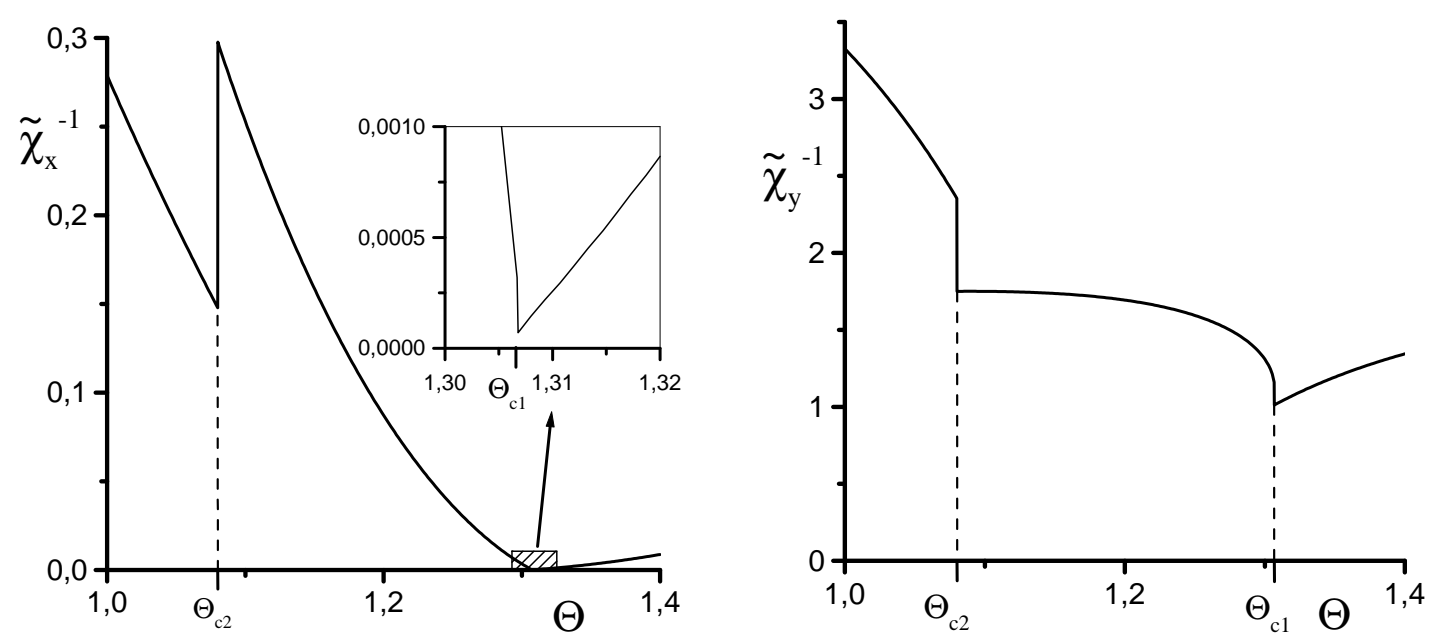

Figure 7: Dependences of components of inverse static dielectrical susceptibility on temperature. Here and in Figure $8 \tilde{\chi}_{\alpha}=a_{1} v_{c} \chi_{\alpha}$.

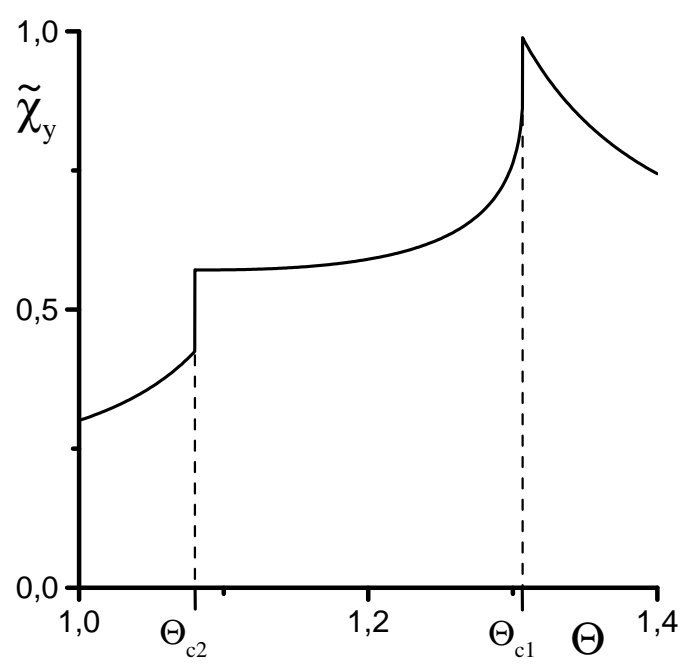

Figure 8: Dependence of the Y component of static dielectrical susceptibility on temperature.

for the DMAGaS crystal but those for the DMAAlS (Kapustianik et al., 1994) close resemble the theoretical picture. Both components have jumps at temperature of the first order phase transition from antiferroelectric to ferroelectric phase.

Basing on the experimental data some approximate evaluation of values of parameters has been made. A value of polarization of saturation $P_{S}=1.8 \cdot 10^{-2} \mathrm{C} / \mathrm{m}^{2}$ gives $d_{x}=6.8 \cdot 10^{-30} \mathrm{C} \cdot \mathrm{m}$ (what corresponds to $0.9 \mathrm{e} \times 0.5 \AA$ ). Taking an approximate value $a_{1}=27.4 \cdot 10^{20} \mathrm{~cm}^{-3}$, we obtain $a_{1} d_{x}^{2}=8.3 \cdot 10^{-3} \mathrm{eV}$ (normalizing factor for $\Theta, \Delta$ and $\Omega$ ) and $a_{1} v_{c}=27.4 \cdot 10^{20} \mathrm{~cm}^{-3} \times 786 \cdot 10^{-24} \mathrm{~cm}^{3}=2.15$ (normalizing factor for the components of $\chi$ ). Hence we get $\Delta=0.013 \mathrm{eV}$ (this value corresponds to the observed ratio of occupancies of positions 1,2 and 3,4 at ambient temperature), $\Omega_{c 2}=40 \mathrm{~cm}^{-1}$ and the maximum value of $\chi_{y}=13$ at the point of the high-temperature phase transition what good coincides with the experiment for DMAAlS. 


\section{Conclusions}

A simple dipole approximation for the four-state model proposed for description of thermodynamics of the DMAGaS-DMAAlS family of crystals is developed. The model exhibits complicated thermodynamical behaviour depending on values of system parameters. At certain values of parameters the model gives the same sequences of phase transitions as found experimentally in DMAGaS and DMAAlS.

Numerical exploration of the model shows that increase of the orientational hopping between sites leads to the suppression of ordered phases in the Ising model like way.

An attempt to give theoretical explanation of the experiment on the influence of hydrostatic pressure on phase diagram of DMAGaS crystal results in good qualitative agreement. It is assumed that the main effect of pressure is the change of the difference of site energies.

\section{Acknowledgements}

The authors would like to thank Prof. Z.Czapla and Dr. R.Tchukwinskyi for their interest in the work and useful discussions

This work was supported in part by the Foundation for Fundamental Investigations of Ukrainian Ministry in Affairs of Science and Technology, project No. 2.4/171.

\section{Appendix A. Transformation of the matrix of dipole-dipole interactions to irreducible repre- sentations of the high-temperature symmetry group}

$$
\hat{\psi}=\left(\begin{array}{cccccc}
a^{\prime} & b^{\prime} & c^{\prime} & d^{\prime} & e^{\prime} & f^{\prime} \\
b^{\prime} & g^{\prime} & h^{\prime} & -e^{\prime} & k^{\prime} & l^{\prime} \\
c^{\prime} & h^{\prime} & m^{\prime} & f^{\prime} & -l^{\prime} & n^{\prime} \\
d^{\prime} & -e^{\prime} & f^{\prime} & a^{\prime} & -b^{\prime} & c^{\prime} \\
e^{\prime} & k^{\prime} & -l^{\prime} & -b^{\prime} & g^{\prime} & -h^{\prime} \\
f^{\prime} & l^{\prime} & n^{\prime} & c^{\prime} & -h^{\prime} & m^{\prime}
\end{array}\right) .
$$

Here axis indices are changed first (e.g. $\{k \alpha\}=\{1 x, 1 y, 1 z, 2 x, 2 y, 2 z\})$.

Matrix of unitary transformation is as follows:

$$
\hat{U}=\frac{1}{\sqrt{2}}\left(\begin{array}{cccccc}
1 & 0 & 0 & -1 & 0 & 0 \\
0 & 1 & 0 & 0 & 1 & 0 \\
0 & 0 & 1 & 0 & 0 & -1 \\
1 & 0 & 0 & 1 & 0 & 0 \\
0 & 1 & 0 & 0 & 1 & 0 \\
0 & 0 & 1 & 0 & 0 & 1
\end{array}\right) .
$$


After the transformation the matrix of interactions

$$
\begin{aligned}
& \hat{\tilde{\psi}}=\frac{1}{2}\left(\begin{array}{cccccc}
a^{\prime}-d^{\prime} & b^{\prime}+e^{\prime} & c^{\prime}-f^{\prime} & 0 & 0 & 0 \\
b^{\prime}+e^{\prime} & g^{\prime}+k^{\prime} & h^{\prime}-l^{\prime} & 0 & 0 & 0 \\
c^{\prime}-f^{\prime} & h^{\prime}-l^{\prime} & m^{\prime}-n^{\prime} & 0 & 0 & 0 \\
0 & 0 & 0 & a^{\prime}+d^{\prime} & b^{\prime}-e^{\prime} & c^{\prime}+f^{\prime} \\
0 & 0 & 0 & b^{\prime}-e^{\prime} & g^{\prime}-k^{\prime} & h^{\prime}+l^{\prime} \\
0 & 0 & 0 & c^{\prime}+f^{\prime} & h^{\prime}+l^{\prime} & n^{\prime}+m^{\prime}
\end{array}\right) \\
&=\left(\begin{array}{cccccc}
a_{1} & b_{1} & c_{1} & 0 & 0 & 0 \\
b_{1} & d_{1} & e_{1} & 0 & 0 & 0 \\
c_{1} & e_{1} & f_{1} & 0 & 0 & 0 \\
0 & 0 & 0 & a_{2} & b_{2} & c_{2} \\
0 & 0 & 0 & b_{2} & d_{2} & e_{2} \\
0 & 0 & 0 & c_{2} & e_{2} & f_{2}
\end{array}\right)
\end{aligned}
$$

becomes block-diagonal, where the upper left block corresponds to the $A_{u}$ representation and the bottom right one to the $B_{u}$.

\section{References}

Dacko S. and Z. Czapla (1996). On the phase transitions in DMAAS and DMAGaS, Ferroelectrics, 185, 143.

Dolinšek J., M. Klanjšek, D. Arčon, Hae Jin Kim, J. Selinger, V. Žagar and L.F. Kirpichnikova (1999). ${ }^{1} \mathrm{H}$ and ${ }^{27} \mathrm{Al}$ study of the ferroelectric transition in dimethylammonium aluminum sulphate hexahydrate $\left(\mathrm{CH}_{3}\right)_{2} \mathrm{NH}_{2} \mathrm{Al}\left(\mathrm{SO}_{4}\right)_{2}$. $6 \mathrm{H}_{2} \mathrm{O}$, Phys. Rev. B, 59, 3460 .

Kapustianik V., M. Bublyk, I. Polovinko, S. Sveleba, Z. Trybula and E. Andreev (1994). Dielectric properties of $\mathrm{NH}_{2}\left(\mathrm{CH}_{3}\right)_{2} \mathrm{Al}\left(\mathrm{SO}_{4}\right)_{2} \cdot 6 \mathrm{H}_{2} \mathrm{O}$ crystals at low temperatures, Phase Transitions, 49, 231.

Kazimirov V.Yu., E.E. Rieder, V.A. Sarin, A.V. Belushkin, L.A. Shuvalov, L.E. Fykin and C. Ritter (1998). Neutron structural investigations of phased transition in DMAAS crystals, J. Korean Phys. Soc., 32, S91.

Pietraszko A. and K. Łukaszewicz (1994). Crystal structure of $\left(\mathrm{CH}_{3}\right)_{2} \mathrm{NH}_{2} \mathrm{Al}\left(\mathrm{SO}_{4}\right)_{2} \cdot 6 \mathrm{H}_{2} \mathrm{O}$ in the low temperature ferroelectric phase, Polish J. Chem., 68, 1239.

Pietraszko A., K. Łukaszewicz and L.F. Kirpichnikova (1993). Crystal structures of $\left(\mathrm{CH}_{3}\right)_{2} \mathrm{NH}_{2} \mathrm{Al}\left(\mathrm{SO}_{4}\right)_{2} \cdot 6 \mathrm{H}_{2} \mathrm{O},\left(\mathrm{CH}_{3}\right)_{2} \mathrm{NH}_{2} \mathrm{Ga}\left(\mathrm{SO}_{4}\right)_{2} \cdot 6 \mathrm{H}_{2} \mathrm{O}$, and $\left(\mathrm{CH}_{3}\right)_{2} \mathrm{NH}_{2} \mathrm{Al}\left(\mathrm{S}_{0.89} \mathrm{Se}_{0.11} \mathrm{O}_{4}\right)_{2} \cdot 6 \mathrm{H}_{2} \mathrm{O}$, Polish J. Chem., 67, 1877.

Pietraszko A., K. Łukaszewicz and L.F. Kirpichnikova (1995). Crystal structure of $\left(\mathrm{CH}_{3}\right)_{2} \mathrm{NH}_{2} \mathrm{Ga}\left(\mathrm{SO}_{4}\right)_{2} \cdot 6 \mathrm{H}_{2} \mathrm{O}$ in the ferroelectric phase at $125 \mathrm{~K}$ and low temperature phase at $100 \mathrm{~K}$, Polish J. Chem., 69, 922. 
Pykacz H. and Z. Czapla (1997). Dielectric and dilatometric studies of $\left(\mathrm{CH}_{3}\right)_{2} \mathrm{NH}_{2} \mathrm{Ga}\left(\mathrm{SO}_{4}\right)_{2} \cdot 6 \mathrm{H}_{2} \mathrm{O}$ (DMAGaS), Ferroelectrics Letters, 22, 107.

Sobiestinskas P., Y. Grigas, E.F. Andreev and E.M. Varikash (1992). Phase Transitions, 40, 85 .

Stasyuk I.V. and O.V. Velychko (2000). Microscopic model of phase transition in the crystals of DMAAlS and DMAGaS type, Journ. Phys. Studies (in print); Preprint cond-mat/9912281, www.http://arXiv.org/abs/cond-mat/9912281

Stasyuk I.V., O.V. Velychko, Z. Czapla and R. Czukwinski (2000). Thermodynamics and dielectric anomalies of DMAAS and DMAGaS crystals in the phase transitions region (Landau theory approach), Condensed Matter Physics, 3 (in print); Preprint cond-mat/0001156, www.http://arXiv.org/abs/condmat/0001156

Tchukvinskyi R., R. Cach and Z. Czapla (1998). Dielectric study of the ferroelectric phase transition in DMAGaS crystal, Z. Naturforsch., 53a, 105.

Yasuda N., A. Kaneda and Z. Czapla (1999). Pressure effects in dimethylammonium gallium sulfate (DMAGaS), Ferroelectrics, 223, 71. 